\title{
Comparative Study on Performance Appraisal System in American and Chinese Enterprises
}

\author{
Shuyu Zhou ${ }^{1, *}$ \\ ${ }^{1}$ Department of Business, Beijing Technology and Business University, Beijing 100037, China \\ *Corresponding author. Email: 13568110970@163.com
}

\begin{abstract}
The performance management is important in corporate governance, and performance appraisal is the major element in performance management. By learning the prevailing key performance indicators method and balanced score card appraisal method in American enterprises, and exploring the development of Chinese performance appraisal in three different stages, this article demonstrates the features of performance appraisal in each stage and looks into the inherent reasons behind the differences between the enterprises in America and China. It also provides guidance on what improvement can be made to construct a more efficient appraisal system in Chinese corporations. By giving advice on the appraisal target, process and culture, a comprehensive and long-term appraisal system can be developed and the governance can be promoted. This article provides suggestions on appraisal system of Chinese enterprises for further development.
\end{abstract}

Keywords: Comparative study, Performance appraisal, Key performance indicators, Balanced score card, The US and Chinese enterprises.

\section{INTRODUCTION}

One of the major trends in modern industry is the prosperity of the service industry, which requires better human resource management. As the most important part of the service industry, human resources are essential to enterprises. It is also essential to establish a reasonable and appropriate evaluation system[1]. And it is more difficult to evaluate performance of the corporation that provides the service, because the delivered products are more complex and sometimes invisible. In addition, under the prevailing trend of globalization, Chinese enterprises need to step out and international enterprises need to enter Chinese market. The interactions between the two largest economy entities, the US and China, are inevitable. That is the opportunity when Chinese enterprises can learn from the US and adapt themselves into international market. To ensure the core competitive advantages, Chinese corporations must establish a better constructed appraisal system[2].

To construct a more suitable appraisal system, it is necessary to learn key performance indicators and balanced score card in the US, and then explore Chinese appraisal standard evolvement in three different stages, and find the features of each stage. By comparing the two different systems, the inherent reasons of the difference can be explained. By finding the roots of disadvantages and differences, the improvements can be made in each aspect. There is no universal appraisal system that suits every entity, so Chinese corporations need to adjust the system for different environment, and connect the strategic goal with external environment.

\section{PERFORMANCE APPRAISAL IN AMERICAN ENTERPRISES}

There are two prevailing performance appraisal methods in the US, which are key performance indicator (KPI) and balanced score card (BSC). Both of these two well-developed methods provide guidance to guarantee a sustainable development. The BSC method is also popular in some European countries while KPI is mainly applied in the US.

\subsection{Key Performance Indicator}

The content of KPI in each industry is different, as shown in "Table 1". 
Table 1. Different content of KPI in various industries

\begin{tabular}{|l|l|}
\hline \multicolumn{1}{|c|}{ Various Industries } & \multicolumn{1}{c|}{ The content of KPI } \\
\hline Construction industry & Performance completion rate, accident, project acceptance, budget achieving rate. \\
\hline Manufacture industry & Defective rate, idle time, receivable days, attendance rate \\
\hline Insurance industry & $\begin{array}{l}\text { First year premium achievement rate, } 13 \text { month continuance rate, drop rate, } \\
\text { Increase rate, per capita premium, per capita unit }\end{array}$ \\
\hline Advertisement industry & Efficiency of advertising, visibility, ad conversion rate \\
\hline
\end{tabular}

Key performance indicator is a performance appraisal method which starts with analyzing the core successful factors of a corporation's strategic success, and then identifying the key performance indicators which plays a critical role in creating value and taking them as standard for performance measurement. It demonstrates the Pareto Principle, suggesting that enterprises should focus on $20 \%$ core activities which ultimately affect the long term success. It creates a new way of management by connecting the measurement of key indicators with business core activity, by which employee and corporation's goal are closely allied[3].

The KPI setting process is to break the major goal into smaller pieces and allocate them to individuals from the large entity. The first level is the strategic level, which starts from highest level by looking at long-term strategy and the external environment. The second level is tactical level which falls on department tasks. It assigns the responsibility and aims at department level by interpreting the indicators of strategic level. And the last level is about individual position KPI, allocating the department level goals to each position to ensure the consistency with the whole KPI structure.

There are some advantages about KPI. The first advantage about KPI is that it is clear and motivating. By setting specific targets and providing clear guidance, it helps the employees to understand the priority and importance among massive work, and facilitate the upper level to allocate resource accordingly. Second advantage is about the communication benefits. Employees can communicate at any time and provide assistance before and during phased control, so as to ensure more stable future results. The third benefit is about the flexibility as the KPI can be adjusted when the internal or external environment has changed. And as it is timely, it is easy to spot the gap between budget and current situation.

However, there are some problems about applying KPI method in reality. The first problem is about the quantifying indicators. It can be hard to implement qualitative indicators as it involves subjectivity and discretion, but not all indicators can be quantified, which brings barriers in implementing KPI in a corporation. The second problem is about the results-oriented management. Because KPI emphasizes the final results, it may ignore some problems during process and lead to omission of dynamic changes. The third problem is high requirement about management skill as the first step of KPI setting starts at the highest level, and it is mainly decided by the management team. A small mistake in the strategic level could deliver wrong information about the value creating.

\subsection{Balanced Score Card}

Just as KPI in different industries, BSC also evaluates the performance of employees from various angles, in different industries, as is shown in "Table 2".

Table 2. Different content of BSC in various industries

\begin{tabular}{|l|l|}
\hline \multicolumn{1}{|c|}{ Different industries } & \multicolumn{1}{c|}{ The content of BSC } \\
\hline Finance & Return on capital, cash flows, sales growth \\
\hline Internal business process & New product develop, cost control, qualification rate \\
\hline Learning and growth & Employee training, innovation, knowledge asset \\
\hline Customer & Customer satisfaction, retention rate, market share \\
\hline
\end{tabular}

The balanced score card uses four aspects to appraise, and assign different weight to the various aspects according to the strategic need of entity. They contain elements of both internal and external, financial and non-financial, and long-term and short-term aspect, thereby giving a more complete and accurate judgement[4]. Besides the four aspects, the balanced score card is different from the KPI method. It assigns different weights to the four aspects according to the needs in different environments[5]. These four aspects can be 
emphasized separately, but they are essentially interconnected and mutually supportive.

The process about applying the balanced score card is similar to the KPI setting process, from the strategic level to lower level. The difference about the balanced score card is that it sets the targets from four major goals. The financial aspect considers the shareholders' interest, which is mainly measured by profitability. In the aspect of customers, it takes how customers feel into consideration and aims to improve satisfaction. It also concerns indicators about customer needs and markets share. The internal business process is mainly about the operation efficiency to maximize the output with limited resources, concerning indicators in daily operation. In order to ensure long-term success, the company should provide employees with career guidance to provide opportunities for future development.

There are some advantages about BSC. The first advantage is that the four aspects are the causes and results of each other. They are complete and consistent. Compared to KPI and other traditional methods, it concentrates on non-financial information as well as long-term indicators, thereby providing a more reasonable structure. The second advantage is that it considers the inherent causes for one specific indicator. Each indicator belongs to one aspect, so it is easy to spot the weakness in four aspects.

However, the first disadvantage is about the cost. The application of the balanced score card is complicated in reality as it requires massive information from different aspects and high coordinate of management skills, so it might only be feasible in large enterprises instead of medium and small size entities. Another disadvantage is similar with the KPI problem for quantify indicators. BSC contains more information about qualitative requirements, so it is affected to subjective influence and loses objectivity.

\section{PERFORMANCE APPRAISAL IN CHINESE ENTERPRISES}

\subsection{Introduction About Chinese Appraisal System in Three Stages}

In the planned economy stage of early fifties, Chinese central government initiates a series of construction and develop actions to resurge national economy. The state-owned enterprises prosper especially the heavy industry. To assist performance appraisal under special condition, China establishes a united enterprise planning management system, which means the enterprises produce as the order from government. At that time, the production is the key performance indicator. During the period between the planned economy and the opening up policy, the performance evaluation is temporarily canceled, because it is considered to be profit-oriented and contrary to the policy of institutional revolution, but after that tumble period, government publishes another set of appraisal indicators which still focuses on production with additional requirement about quality and profit.

During the reform and opening up period, the efficiency has come to light. With delegation and opening up policies, enterprises operation becomes more flexible and discretional. Then the government focuses on profit and tax contribution, especially when the contract system prevails, which is a form of management delegation and separation of ownership and management. With the development of discretion scope and market maturity, government comes up with indicators related to more aspects, such as sales growth, cash cycle, and resource consumption. However, since the contract system plays a significant role, the disordered management makes practice of measurement in reality a problem.

In the stage of deepening the reform and construction of the modern enterprise system, in order to complete the socialist market economy and modern enterprise system, the performance appraisal indicators contain a more complete vision, including debts payment, operation efficiency and profitability. And then after a series of amendments, the modern enterprise appraisal system concentrates on the entities' capital efficiency, capital management, debts payment and development ability.

There are some features of the Chinese appraisal system. The first trait of Chinese enterprises performance appraisal comes from different market structure, in which the state owned enterprises starts as a leading role. So appraisal standard in the market are mainly designed by the central government to construct a consistent system for performance evaluation, and to give power to government in standard setting. The second characteristic is about the appraisal development, which is heavily influenced by requirement of 
different time stage. Because of a late start of performance appraisal exploration, China focuses on results to meet a time need, such as production and profit, so the change of performance appraisal standard is driven by time need. The third function is about information users. In order to ensure the national interest, they have conducted supervision and management evaluation. Therefore, its focus on production and sustainability is more limited.

\subsection{Advantages and Disadvantages in Chinese Performance Appraisal}

The first advantage is that the appraisal is target driven, so in immature markets (such as China in the 1950s), evaluation is straightforward and practical, and it gives a clear and focused direction. Secondly, it provides consistency among companies, making it easier to evaluate all of them from the center. At the same time, it is obvious to spot the difference between completion and target, which can provide a clear gap.

However, for state-owned enterprises, the focus of performance appraisal is limited to the standard set by government, which impedes evaluations from other perspective. As a result, the concern of creditors, small and medium size shareholder, institutional investors have not gained adequate attention. This also affects the corporations' motivation and even misses the opportunities in capital market. Besides, the corporations are mainly appraised by the government officer and business principal, and the basic goal is to meet the set target, which is not proactive, and this will lead to potential efficiency problems. When the budget cost is overestimated, state-owned enterprises tend to create large expenditures to utilize the original budget. In a word, the universal system omits the characteristics and concerns of entities and other individuals. As a result, short-sighted vision and opportunism also appear to be a problem.

For civilian-run enterprises, they may lack of standardization and efficiency because of misunderstanding about performance appraisal. Most of the civilian-run enterprises tend to be small and medium-sized, but they play an active role in market with rapid expansion. It is important to realize their shortcomings. The performance appraisal system tends to be more flexible, and lack regulation and requirement. Some of the enterprises prefer to evaluate operational indicators, such as job qualification, and significant transaction performance, because they are direct and easily obtained. However, these evaluations are too specific and rely on human judgement, lacking connection with strategy[6]. Because of the high cost of establishing complete evaluation system, the problem of human subjectivity, myopia and high reliance on managers' personal skills tend to be the main concern in civilian-run enterprises, which is why personal management style has a stronger influence on civilian-run enterprises[6].

\section{COMPARISON OF PERFORMANCE APPRAISAL IN AMERICAN AND CHINESE ENTERPRISES}

\subsection{Differences Between the Performance Appraisal in American and Chinese Enterprises}

\subsubsection{Differences in Setting Purpose}

In the American system, the appraisal process aims to improve management efficiency and skill development, to ensure sustainability and long term success. For example, the balanced score card method considers the innovation and learning process as one evaluation goal, which can help managers to manage from long-term perspective. In American corporations, the development of corporations and individual employees are both important and they promote goal congruence between the two. So it is reasonable and critical for corporation to provide adequate employee training and improve management process.

In Chinese corporations, the aim of appraisal goal setting is basically set the target, especially for state-owned enterprises, and then the corporation try to meet the set goal in planned economy stage. Although China accomplishes the transfer from planned economy to market economy, the goal setting is a top-down method as a mean to achieve task from upper level. The appraisal goal literally set the targets the corporations have to reach. It is more task-oriented, so the future perspective and employee development can't obtain adequate attention unless they are proved to be critical in achieving the set target.

This kind of difference in appraisal goal makes the appraisal process affect employees' motivation and operation efficiency. Because of task given system in Chinese corporations, there are less involvement and negotiation in appraisal standard in lower level. The goal setting process highly relies on management skill and given strategy. This 
task-oriented system might be direct in task giving; however, it may have negative effect on floor staff, and affect long-term success.

\subsubsection{Differences in Setting Methods}

The goal of appraisal setting is to facilitate corporations' development in accordance with strategy. Besides the KPI and BSC used in specific appraisal setting process, the American corporations firstly apply the SWOT analysis to corporations' current situation to know about their strength, weakness, opportunities and threats. Secondly, corporations will set target for the next year after developing strategic goal. Thirdly, it is the HR department to divide the major goal into tasks for each department and individuals by various technologies, and clarifies the obligations and duties. Only this way can the companies create a reasonable and feasible target[7].

Chinese enterprises have similarity to the US corporations in setting target and goal for the next year before the current year ends. But the process is more like an up-down task giving, lacking sufficient formal analysis, especially for small and medium sized enterprises. The traditional Chinese style tends to gather the core personnel in a leisure place, and discuss about current year performance evaluation and next year's target. Then the subordinate department will visualize the general goal by producing specific tasks.

US method in appraisal target setting is more strict and reasonable. It produces a feasible and plausible target. The difference between target settings is up to the culture trait. The US companies prefer data and information fact, while Chinese corporations emphasize the personal effect. Chinese corporations tend to be affected by personal style of the leaders, and make decisions based on the personal experience and instinctual guidance, which is actually considered to be one of the key elements in corporation success.

\subsection{Advice for Appraisal System in China}

\subsubsection{Appraisal Indicator Setting}

The first step is to set right indicators. No matter what method applied in goal setting, the indicators have to meet the SMART rules, being specific, measurable, attainable, relevant and time- based. The goal has to be tightly combined with the five requirements to ensure effectiveness.

Secondly, the indicators should also be adjusted when there is a gap between budget and reality. Chinese enterprises tend to conduct performance evaluation at the end of the year, but necessary adjustment and guidance during the year is also important. Exploring the reasons and providing recommendation or adjusting the set target to adapt to the changing environment are two ways to solve this problem.

Thirdly, as the current appraisal goal in China is mainly profit-oriented and major shareholder concerned, other entities' right and interest are not adequately emphasized. The indicators should also contain other perspectives to guarantee a long-term success, and the enterprise's social responsibility in Apple inc. is a good example.

In general, the basic problem in Chinese corporations' goal setting is that they are rigid and partial. This is due to the historic reasons in planned economy, lacking flexibility and communication in execution at the same time. In current fast changing environment, the goal should not be one side decided and stay constant during the whole process. And the concerns of upstream, downstream, society and other institutions are critical for future sustainability. That's why a more complete and comprehensive indicators setting model is needed.

\subsubsection{Improving Appraisal Process}

The whole appraisal process in China is decided by the minority, and then they evaluate subordinates' performance by comparing the target and reality. This just gives the basic view of the differences, and this process is not related to management and efficiency improvement. The interactions with the subordinates are critical in developing effective appraisal system. The appraisal is not a single view flow from up to bottom; instead, it is a series of interaction and communication in the corporation. To make it more efficient, it is important to know the appraisal objective's need. After the appraisal, besides rewards and punishment, follow-up training and adjustments should be considered to construct a more efficient human resource structure. The appraisal is not the result itself, but a mean to improve corporate governance. 


\subsubsection{Establishing Appropriate Culture}

Unlike the active participation culture in the US working environment, where employee individuals are highly valued and respected, Chinese corporations emphasize the leaders' personal effect. To some extent, this lead to a tame atmosphere in corporations for employee individuals, and this is not good for participation and involvement. One important goal of performance evaluation is to motivate employee to progress towards strategic direction. Besides this, employees' personal capability is also important in modern service industries. So the corporations need to create their own culture which meets the criteria to motivate employees to demonstrate their own thoughts and minds, and then be willing to involve in the operations.

Besides the common requirement of motivations and participation, to construct appropriate corporation culture, the corporations need to evaluate its strategy, and take the environment and internal resources into account, especially HR. After allying the culture with strategy and embedding culture in employees' mind, they will automatically adjust their personal behavior according to the strategy. When employee has the intention to ally their daily conduct with corporation's strategy, the whole corporation's performance can be improved, and the results of appraisal will also be more satisfactory.

\section{CONCLUSION}

For companies, it is very important to build an effective evaluation system to track the performance of entities and to derive future significance from the evaluation results. The KPI and BSC methods in America have been well developed and have performed well in terms of goal consistency and sustainability. Both of the two methods, however, have disadvantage of quantifying practice, which is not easy in real appliance. As for Chinese enterprises, they are heavily affected by how history evolves and maintaining some native culture features. For stateowned enterprises, the appraisal system in China can meet the time need and focus on both users and the government. Although the requirement in modern China emphasizes future development, corporations still need further instructions on how to construct a system that adapts to the modern dynamic environment with future orientation. For the civilian-run enterprises, the problem is mainly about unregulated methods and heavy cost to establish a complete system like BCS, so they prefer to the use simple indicators in operation level. To build a more efficient appraisal system, China should set appropriate goal and promote process instruction to ensure the effective realization of strategic goals and a culture that encourages all employees to participate. The subjective judgment of China's assessment shows how Chinese culture values the connections between people and organizations, but companies need to limit this influence to avoid extreme and shortsightedness. Further development requires enterprises to figure out suitable, acceptable and feasible structure itself and then proactively evolve towards the goal of constructing a well-developed system.

\section{AUTHORS' CONTRIBUTIONS}

This paper is independently completed by Shuyu Zhou.

\section{REFERENCES}

[1] Wu Xiaoxia, The establishment and implementation of enterprise performance evaluation system and incentive system, Human Resources, vol 18, 2020: 126-127.

[2] Pei Quan, Yong Shi, Lingfeng Niu, et al, Automatic Chinese Multiple-Choice Question Generation for Human Resource Performance Appraisal, Procedia Computer Science, vol 139, 2018:165-172.

[3] Wen Subin, Guo Yubing, Key performance Indicator method: Interpretation and application cases, Friends of Accounting, vol 19, 2020: 148-153.

[4] Tong Mengjie, Capitalization of intangible assets, performance evaluation of nonfinancial indicators and corporation financial performance, Communication of Finance and Accounting, vol 18, 2020: 35-38.

[5] Tang Xiangyang, An analysis of balanced score card application in enterprises, Money China, vol 25, 2020: 59-60.

[6] Ye Zhen, A brief analysis of the problems and countermeasures of performance management in small and medium-sized private enterprises, China Collective Economy, vol 26, 2020: 118119. 
[7] Liu Chen, A brief discussion on the problems existing in the internal performance evaluation of enterprises and their solutions, Modern Business, vol 25, 2020: 106-107.

[8] Muhammad Zahid Iqbal, Saeed Akbar, Pawan Budhwar, et al, Effectiveness of performance appraisal: Evidence on the utilization criteria, Journal of Business Research, vol 101, 2019: 285-299. 